\title{
Compressive Sensing for Multi-Static Scattering Analysis
}

\author{
Lawrence Carin, Dehong Liu, Wenbin Lin and Bin Guo \\ Department of Electrical and Computer Engineering \\ Duke University \\ Durham, NC 27708-0291 \\ \{lcarin,liudh,wl,bg25\}@ece.duke.edu
}

\begin{abstract}
Compressive sensing (CS) is a framework in which one attempts to measure a signal in a compressive mode, implying that fewer total measurements are required vis-à-vis direct sampling methods. Compressive sensing exploits the fact that the signal of interest is compressible in some basis, and the CS measurements correspond to projections (typically random projections) performed on the basisfunction coefficients. In this paper we demonstrate that when a target is situated in the presence of a complicated background medium, the frequency-dependent multi-static fields scattered from the target may be measured in a CS manner; the CS measurements are performed by exploiting the natural wave propagation of the incident and scattered fields. This phenomenon is related to the field of time reversal, to which relationships are made. We also demonstrate that the CS framework may be employed to reduce the number of computations required in numerical linear scattering analyses. Example scattering results are presented for multi-static electromagnetic scattering, and application areas are also discussed.
\end{abstract}

\section{INTRODUCTION}

In the 1990s there were significant developments on the sparse representation of digital signals in terms of orthonormal basis functions. For example, assume that one is interested in representing the $m$-dimensional real signal $u$ in terms of a basis, of the form $u=\Psi \theta$, where $\Psi$ is an $m \times m$ real matrix and $\theta$ represents an $m$-dimensional column vector; the columns of $\Psi$ represent the $m$ orthonormal basis vectors. For most natural signals $u$ (characterized by being piecewise smooth), the wavelet transform [1], [2] has been shown to yield a particularly sparse representation. Specifically, if $\theta$ represents the wavelet coefficients for a piecewise-smooth signal $u$, then the error $\left\|\theta-\theta_{N}\right\|_{2}^{2}$ has been shown to decay quickly with increasing $N$, where $\theta_{N}$ represents $\theta$ 
with the smallest $m-N$ coefficients set to zero. This implies that only a small number $N$ of dominant wavelet coefficients are required to approximate $u$ well. This property of wavelets has led to a new generation of wavelet-based compression algorithms, for both imagery and video [3], [4]. Importantly, since the $N$ dominant wavelet coefficients are a strong function of the signal $u$ of interest, the aforementioned compression algorithms must adapt to $u$.

While wavelet-based compression and sparse-signal representations have had many important practical applications, in the context of sensing there are issues worthy of further research. For example, in the above discussion it was noted that the $m-N$ smallest wavelet coefficients may be discarded with minimal degradation to the reconstruction of $u$ (i.e., $\left\|\theta-\theta_{N}\right\|_{2}^{2}$ decreases quickly as $N$ increases). However, in the context of sensing, one must first measure the $m$ dimensional digital signal $u$, represent it in the wavelet basis $\Psi$, and then compression is performed subsequently, after which effectively $\theta_{N}$ is retained. Consequently, in some sense $m-N$ pieces of data in the original $u$ were measured unnecessarily. Compressive sensing (CS) ${ }^{1}$ [5]-[7] is a new approach to data collection, seeking to integrate sensing and compression and therefore measure the informative part of the signal directly. The above discussion was placed in the context of wavelets, while CS is applicable to any basis or frame $\Psi$ in which the signal of interest $u$ may be sparsely rendered [5]-[7]. For example, in some of the example results presented below, we will utilize a discrete cosine transform rather than the wavelet transform.

There are several "surprising" aspects of compressive sensing [7]. First, in the above discussion it was noted that the largest $N$ coefficients $\theta_{N}$ are a strong function of the signal $u$, and the approximation to $u$ based on $\theta_{N}$ yields a good representation with $N \ll m$. Therefore, one may anticipate that the CS measurements should be performed adaptively, with the system "homing in" in some sense on the dominant coefficients $\theta_{N}$. A surprising aspect of CS is that one does not attempt to measure the dominant coefficients $\theta_{N}$; rather, a weighted combination of all wavelet coefficients $\theta$ are measured. Another surprising aspect of CS is that one may expect that the aforementioned weights should adapt to the signal under test; however, CS theory indicates that a fixed set of weights may be used for all $u$ within a given class, and that the quality of the CS estimate of $u$, based on these measurements, will not be significantly worse than the best adaptive measurements. A set of $n$ CS measurements may be represented as $v=\Phi \theta$, where $\Phi$ is an $n \times m$

\footnotetext{
${ }^{1}$ All compressive-sensing preprints in the list of references may be downloaded from http://www.dsp.ece.rice.edu/cs/
} 
projection matrix. One additional "surprise" is that the components of $\Phi$ may be constituted as draws from a general underlying random variable. Summarizing, (i) in CS measurements one does not directly measure $u$, but measures $u$ (or, equivalently, $\theta$ ) projected onto a random signal, and $n$ such random projections constitute the $n$-dimensional CS measurement vector $v$; (ii) the particular $n$ projection vectors may be constituted randomly, and are fixed for all $u$; (iii) one may use CS inversion techniques to approximate $u$ accurately based on the measurements $v$, with several techniques available for this inversion [6], [8]-[13].

In this paper we address the use of CS to perform compressive wave-scattering measurements, building upon the CS ideas summarized above. For simplicity we first consider two-dimensional scattering, as a function of the angle $\phi$, but the basic approach is applicable to three-dimensional scattering problems. Assume that the signal of interest is represented as $u_{s}\left(\phi_{i}, \phi_{s}, \omega\right)$, constituting the far-zone fields scattered from a general target; $u_{s}\left(\phi_{i}, \phi_{s}, \omega\right)$ corresponds to planewave excitation at angle $\phi_{i}$, with the scattered fields observed at scattering angle $\phi_{s}$, with the measurement performed at frequency $\omega$. Such measurements are typically performed by placing source and receiver antennas in the far zone of the target, and varying the angles $\phi_{i}$ and $\phi_{s}$. Assuming fixed frequency $\omega$, if one considers $\sqrt{m / 2}$ discrete angles $\phi_{i}$ and $\sqrt{m / 2}$ discrete $\phi_{s}$, with $\sqrt{m / 2}$ an integer, then the discretized "image" of data $u_{s}\left(\phi_{i}, \phi_{s}, \omega\right)$ constitutes an $\sqrt{m / 2} \times \sqrt{m / 2}$ complex matrix, and an "unwrapped" representation of this matrix constitutes an $m / 2$-dimensional complex vector $\tilde{u}_{s}$. Since current CS theory applies to real signals, the vector of interest in CS is $u_{s}$, which corresponds to $\tilde{u}_{s}$ with the real and imaginary parts concatenated. In the work that follows CS will be used to estimate the spectral-domain version of the currents induced on the target, and the corresponding real $m$-dimensional vector is represented as $u$ (corresponding to discretized spectral angles), with $u$ and $u_{s}$ linearly related to one another. Thus, consistent with the initial discussion of CS above, the desired real signal $u$ is of dimension $m$.

We now assume that the same target is placed in the presence of a complex propagation medium, characterized by significant multi-path, such as that considered in previous time-reversal studies [14]-[16]. As a consequence of this multi-path, a single source may constitute a complex (non-planar) field incident upon the target, and based upon a Fourier analysis of this excitation field, one may show that the incident field corresponds to a superposition of plane waves incident upon the target at a continuum of different propagation angles. As detailed below, the diversity 
of this effectively multi-plane-wave excitation is dictated by the complexity of the background medium, with the excitation diversity increasing with multi-path. Making the connection to the aforementioned CS discussion, note that the excitation fields in the presence of a complex background medium yield a linear combination of plane waves propagating at different angles $\phi_{i}$; if the properties of the background medium are viewed as a draw from a random process [15], then the weights on these plane waves may (in a loose sense) also be viewed as draws from a random process (defined by the background-medium wave propagation). As one considers different locations of the source within the background medium, the aforementioned weights on the excitation plane waves change accordingly.

The fields that scatter from the target, emanating at different angles $\phi_{s}$, propagate through the same background medium; if that medium is sufficiently complicated, waves emitted at multiple angles $\phi_{s}$ make their way to a given receiver position. These properties of the excitation and scattered fields are depicted schematically in Figure 1. Summarizing, a given source in the presence of the background medium yields a weighted set of plane waves traveling at different angles $\phi_{i}$ in the presence of the target, and the induced scattered fields propagate away from the target at multiple angles $\phi_{s}$, and energy from multiple of these scattering angles arrive at a given receiver, with the different received $\phi_{s}$ components weighted by the associated characteristics of the corresponding propagation medium.

As demonstrated explicitly below, fixed source location $\boldsymbol{\rho}_{e}$ and receiver location $\boldsymbol{\rho}_{o}$ yield a scattering measurement observed at $\rho_{o}$ that corresponds to a linear combination of the components of $u$. The weights on this linear relationship are defined by the plane waves induced in the presence of the target by the source at $\rho_{e}$ (these weight with respect to the discrete angle $\phi_{i}$ ), and the scattered plane waves observed by the receiver at $\boldsymbol{\rho}_{o}$ (these yielding weights with respect to the discrete angle $\phi_{s}$ ). If $n / 2$ different combinations of $\boldsymbol{\rho}_{e}$ and $\boldsymbol{\rho}_{o}$ are considered, then these $n / 2$ measurements define the desired $n$-dimensional CS vector of measurements $v$ (where again $v$ is a concatenation of the real and imaginary parts of the measured CS data, since the actual frequency-dependent scattering data are complex).

Assume now that a discretized representation of the $m$-dimensional real vector $u$ may be sparsely rendered in a given basis $\Psi$; by exploiting the propagation complexity naturally manifested by a sophisticated propagation medium surrounding a target of interest, one has the potential to recover the $m$-dimensional discretized form of the multi-scattering signal $u$ using a 
relatively small number of CS measurements $v$, with the number of CS measurements satisfying $n \ll m$. In the above discussion we considered $u\left(\phi_{i}, \phi_{s}, \omega\right)$ at a fixed frequency $\omega$, discretized to form a matrix in dimensions $\phi_{i}$ and $\phi_{s}$. The same CS idea may be used to consider threedimensional discretization in the space $\left(\phi_{i}, \phi_{s}, \omega\right)$, in which $\Psi$ must represent a basis in a three-dimensional space.

We refer to the concept of exploiting the properties of a complex background propagation meeting to perform compressive-sensing-based scattering measurements as in situ CS, because the multi-path medium surrounding the target is directly utilized to constitute the projections $\Phi$ required in the CS framework. In this paper we place the in situ CS framework sketched above on a firm mathematical footing, and show its application to problems of interest for the characterization of the multi-static signature of targets. Before proceeding to that analysis, we make connections to the related field of time-reversal sensing [14]-[16]. In time reversal one also exploits the properties of a complex medium surrounding a target or source of interest; for simplicity, consider a time-domain source. In time reversal, the fields observed at a set of receivers are reversed in time, and synchronously reradiated from the receivers, and the fields focus coherently at the original source location, approximately recreating the original source (assuming a sufficiently complicated background medium). The interesting aspect of this is that, by exploiting a highly multi-pathing environment (complicated background medium), the resolution with which the fields are focused at the source may be much higher than that expected of the same physical antenna aperture situated in vacuum; this process has been referred to as superresolution, with a detailed mathematical analysis found in [15]. This implies that in the presence of a heterogeneous medium, the signals received at the sparse set of receiver antennas contain more information about the source than would exist if the experiment took place in vacuum, and by time reversing and reradiating the fields that information is approximately recovered at the source location. Hence, through exploitation of multipath, a relatively small set of measurements contain an unanticipatedly large amount of source information, that can be recovered by exploiting the propagation-medium complexities upon time-reversing and repropagating.

In the in situ CS framework a similar setting is realized. The relatively small set of $n$ CS measurements constituting $v$ correspond to the relatively small set of antenna positions in timereversal. The superresolution property in time reversal is analogous to the fact that the $m$ dimensional discretized form of $u\left(\phi_{i}, \phi_{s}, \omega\right)$ may be recovered by a relatively small set of $n \ll m$ 

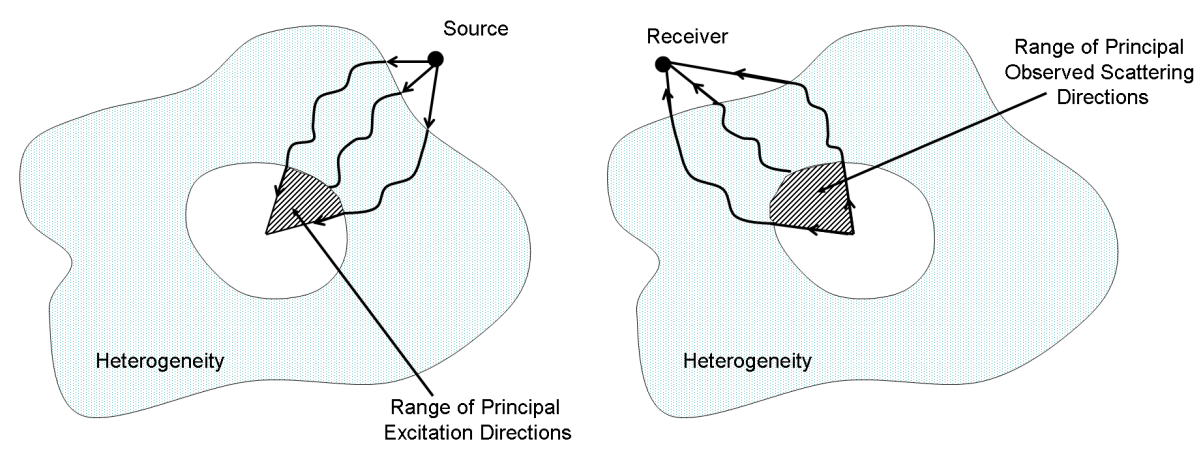

Fig. 1. Depiction of the principal cone of rays contributing to the target excitation by the source (left), and received at the observation point (right). This figure is notional, since in reality not an explicit cone of rays will contribute for either the source or receiver, but rather there will be a weighted continuum of contributing angles, with these corresponding to associated plane waves.

CS measurements $v$. Both time reversal and in situ compressive sensing exploit the complex propagation medium to realize related effects. Time reversal is often performed experimentally, meaning that the measured fields are time-reversed and physically reradiated into the medium, yielding focusing of energy at the original source [14]. The in situ CS framework is interested in recovering the angle- and frequency-dependent scattered fields of a source (radiation) or target (scattering), and in some sense represents a generalization of techniques used in imaging, here explicitly exploiting the complex wave propagation manifested by a complicated background propagation medium.

The remainder of the paper is organized as follows. In Section II we address the scattering of a target embedded in a complex propagation medium, and demonstrate that the scattered fields observed at one point, due to a source at another point, may be interpreted as a CS measurement. Using this concept it is demonstrated how a set of such measurements may be used, in the context of in situ CS, to estimate the angle-dependent fields scattered from the target as a function of plane-wave excitation direction. Applications of this framework are presented in Section III, wherein CS as applied to scattering measurements is examined from two perspectives. Conclusions are discussed in Section IV, wherein further directions and applications are reviewed. 


\section{Scattering From Target Embedded in Complex Medium}

\section{A. Excitation Fields}

Assume we are interested in inferring, through measurements or numerical computations, the multi-static wideband scattering response from a general target. In a traditional such analysis, one considers plane-wave incidence at a set of angles with respect to the target center, and the scattered signal is measured or computed in the far zone as a function of angle, with the target situated in vacuum; such an analysis is typically performed at a sequence of frequencies to obtain the wideband scattering response. When analyzing such a problem in the discussion that follows, we consider the two-dimensional case, thereby simplifying notation, although the basic construct extends naturally to three-dimensional problems. We desire the signal $u_{s}\left(\phi_{i}, \phi_{s}, \rho_{s}, \omega\right)$, which represents the scattered fields of the target when situated in vacuum, with the measurements performed at angular frequency $\omega$, due to a plane wave incident at angle $\phi_{i}$, with the scattered fields observed at angle $\phi_{s}$ (see Figure 2). The scattered fields are assumed to be observed in the far zone, at an arbitrary range $\rho_{s}$.

Concerning notation, $u\left(\phi_{i}, \phi_{s}, \omega\right)$ represents a complex and continuous function of $\left(\phi_{i}, \phi_{s}, \omega\right)$; when this function is discretized with respect to $\left(\phi_{i}, \phi_{s}, \omega\right)$ the corresponding complex vector is represented as $\tilde{u}$. For CS one is interested in real vectors, and the real counterpart of $\tilde{u}$ is denoted $u$, which is a vector twice as long as $\tilde{u}$, with $u$ representing the concatenated real and imaginary parts of $\tilde{u}$. Similar notation is employed for the vector of complex CS measurements $\tilde{v}$, with corresponding real CS measurements $v$. As discussed below, $u\left(\phi_{i}, \phi_{s}, \omega\right)$ will correspond to the Fourier transform of the currents induced on the target of interest, with $\phi_{s}$ representing the spectral angle and $\phi_{i}$ the plane-wave angle of incidence; in the far zone $u_{s}\left(\phi_{i}, \phi_{s}, \rho_{s}, \omega\right)$ is linearly related to $u\left(\phi_{i}, \phi_{s}, \omega\right)$.

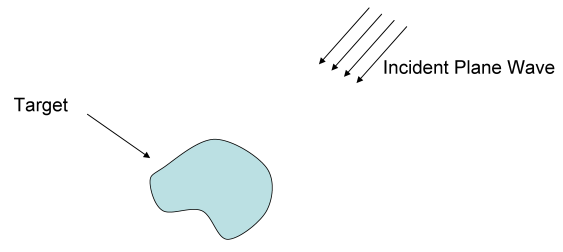

Fig. 2. Schematic of a general target situated in vacuum, excited by a plane wave. 
Rather than performing a scattering analysis in vacuum, as is typical, we assume that the target is surrounded by a complex propagation medium. The objective is to exploit the multipath introduced by such an environment to approximate $u\left(\phi_{i}, \phi_{s}, \omega\right)$ based on a relatively small number of measurements performed in the presence of the heterogeneities, using ideas from compressive sensing; from the estimated $u\left(\phi_{i}, \phi_{s}, \omega\right)$ one may then estimate the far-zone $u_{s}\left(\phi_{i}, \phi_{s}, \rho_{s}, \omega\right)$. Specifically, rather than measuring $u_{s}\left(\phi_{i}, \phi_{s}, \rho_{s}, \omega\right)$ directly, in vacuum, we will perform a relatively small number of measurements in the presence of heterogeneities; the associated data in these measurements will be different from but linearly related to $u_{s}\left(\phi_{i}, \phi_{s}, \rho_{s}, \omega\right)$. Compressivesensing techniques will then be used to invert for $u\left(\phi_{i}, \phi_{s}, \omega\right)$, from which $u_{s}\left(\phi_{i}, \phi_{s}, \rho_{s}, \omega\right)$ follows. The advantage of this indirect approach, as demonstrated in Section III, is that the number of compressive-sensing measurements will be significantly smaller than the number of typical measurements required if the analysts were to be performed in vacuum.

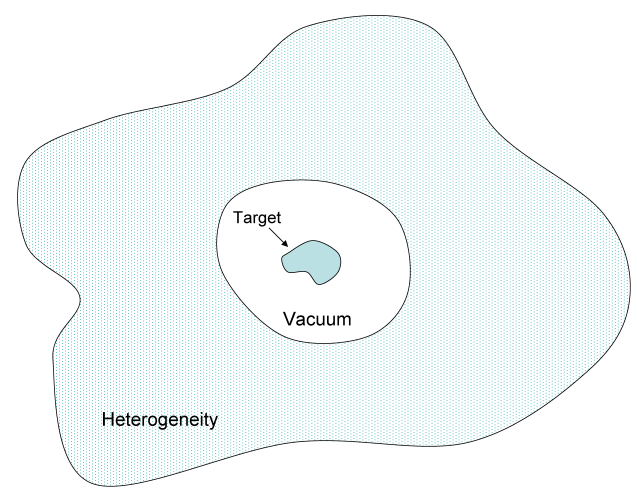

Fig. 3. Schematic of the target in Figure 2 now situated in the presence of a heterogeneous medium. The immediate vicinity of the target is surrounded by vacuum.

In this subsection we discuss how the heterogeneities impact the fields that excite the target, due to a source current situated in the presence of the inhomogeneities (see Figure 3), and in Sections II-B and II-C we discuss how the heterogeneities impact the associated scattered fields. In the discussion that follows we assume the aforementioned excitation is a line current, for analytical simplicity, but the formulation generalizes to arbitrary two-dimensional sources.

In addressing the excitation fields, we consider the source radiating in the presence of the 


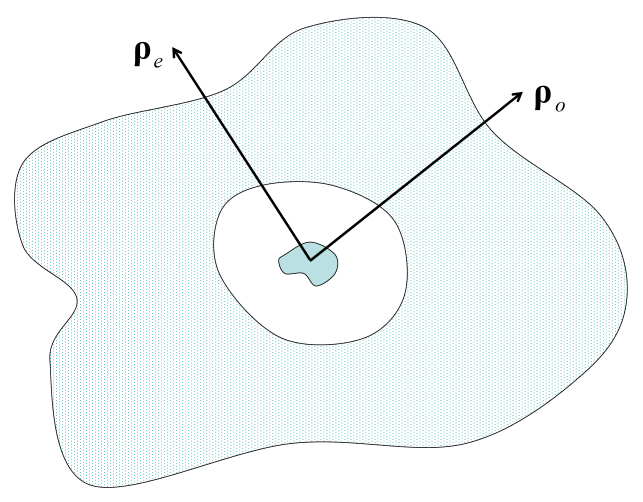

Fig. 4. Location of a line source positioned at $\boldsymbol{\rho}_{e}$, with the scattered fields observed at $\boldsymbol{\rho}_{o}$.

heterogeneous medium, but with the target absent. Specifically, as depicted in Figure 4, assume that a contiguous vacuum pocket is surrounded by a heterogeneous propagation environment, and that a line-source excitation is positioned at arbitrary point $\boldsymbol{\rho}_{e}$ outside the vacuum. As a consequence of the complicated propagation environment, the fields within the vacuum arrive at a wide range of angles (typically a continuum of angles). Assuming that the source is operating at frequency $\omega$, let $g\left(\boldsymbol{\rho}, \boldsymbol{\rho}_{e} ; \omega\right)$ represent the fields at arbitrary position $\boldsymbol{\rho}$ within the vacuum pocket (the coordinate-system origin is at the center of the vacuum); since the source at $\boldsymbol{\rho}_{e}$ is a line source, $g\left(\boldsymbol{\rho}, \boldsymbol{\rho}_{e} ; \omega\right)$ represents the associated two-dimensional Green's function.

We introduce a window function $w\left(\boldsymbol{\rho}, \rho_{t}, \rho_{h}\right)$, with $w\left(|\boldsymbol{\rho}| \leq \rho_{t}, \rho_{t}, \rho_{h}\right)=1, w\left(|\boldsymbol{\rho}| \geq \rho_{h}, \rho_{t}, \rho_{h}\right)=$ 0 , and for $\rho_{t} \leq|\boldsymbol{\rho}| \leq \rho_{h}$ the function $w\left(\boldsymbol{\rho}, \rho_{t}, \rho_{h}\right)$ tapers to zero in an arbitrary smooth manner (see Figure 5). The windowed version of the fields within the vacuum are represented as $g_{w}\left(\boldsymbol{\rho}, \boldsymbol{\rho}_{e} ; \omega\right)=g\left(\boldsymbol{\rho}, \boldsymbol{\rho}_{e} ; \omega\right) w\left(\boldsymbol{\rho}, \rho_{t}, \rho_{h}\right)$.

The two-dimensional Fourier transform of the windowed fields is defined as

$$
\hat{g}_{w}\left(\varphi, \boldsymbol{\rho}_{e} ; \omega\right)=\int_{0}^{\infty} d \rho \int_{0}^{2 \pi} d \phi \rho g_{w}\left(\rho, \phi, \boldsymbol{\rho}_{e} ; \omega\right) \exp \left[j k_{o} \rho \cos (\phi-\varphi)\right]
$$

with $k_{o}=\omega / c, c$ is the wave speed in vacuum, $\varphi$ represents the angle of the spectral component, and the range-angle pair $(\rho, \phi)$ define the vector $\boldsymbol{\rho}$. Physically, $\hat{g}_{w}\left(\varphi, \boldsymbol{\rho}_{e} ; \omega\right)$ represents the amplitude of a plane wave at frequency $\omega=k_{o} c$ propagating in the direction $\varphi$, due to the source 


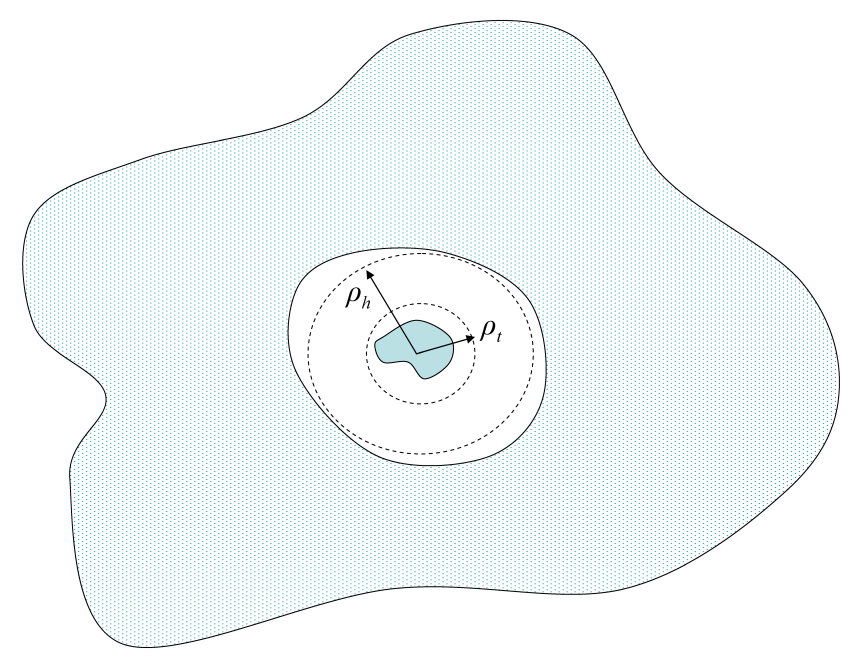

Fig. 5. Depiction of coordinates on window function $w\left(\boldsymbol{\rho}, \rho_{t}, \rho_{h}\right)$.

at $\boldsymbol{\rho}_{e}$, and therefore this is the amplitude of the respective plane-wave component associated with the windowed fields $g_{w}\left(\boldsymbol{\rho}, \boldsymbol{\rho}_{e} ; \omega\right)$. The frequency $\omega$ and source position $\boldsymbol{\rho}_{e}$ are assumed fixed, and therefore $\hat{g}_{w}\left(\varphi, \boldsymbol{\rho}_{e} ; \omega\right)$ is a function of the spectral angle (plane-wave propagation direction) $\varphi$; the diversity of angles $\varphi$ for which $\hat{g}_{w}\left(\varphi, \boldsymbol{\rho}_{e} ; \omega\right)$ has appreciable amplitude, for a line source located at $\boldsymbol{\rho}_{e}$, is dictated by the complexity of the propagation medium (with a wider range of plane waves manifested with increasing background-medium complexity).

Note that for a fixed source, through exploitation of the complex propagation medium, one may effectively constitute excitation plane waves propagating at a continuum of angles in the region within which the target will reside; by contrast, in the absence of the heterogeneity a single propagation direction will dominate, for a fixed source location (assuming the source is distant from the origin). Hence, by exploiting the multipath introduced by the heterogeneities, a single source may introduce a continuum of plane waves incident upon the target, while to achieve the same diversity of excitation for measurements performed in vacuum, a large number of distinct sources must be considered.

The windowed source currents may be recovered via an inverse Fourier transform 


$$
g_{w}\left(\boldsymbol{\rho}, \boldsymbol{\rho}_{e} ; \omega\right)=\frac{k_{o}}{4 \pi^{2}} \int_{0}^{2 \pi} d \varphi \hat{g}_{w}\left(\varphi, \boldsymbol{\rho}_{e} ; \omega\right) \exp \left[-j k_{o} \rho \cos (\phi-\varphi)\right]
$$

assuming that there are no evanescent fields in $g_{w}\left(\boldsymbol{\rho}, \boldsymbol{\rho}_{e} ; \omega\right)$ and hence that $\hat{g}_{w}\left(\varphi, \boldsymbol{\rho}_{e} ; \omega\right)$ exists in the spectral domain on a circle of radius $k_{o}$. The expression in (2) underscores that the spectral form of the source excitation $\hat{g}_{w}\left(\varphi, \boldsymbol{\rho}_{e} ; \omega\right)$ is only a function of the angle $\varphi$. Thus motivated, it will prove convenient in Section II-D to constitute a discretized form of $\hat{g}_{w}\left(\varphi, \boldsymbol{\rho}_{e} ; \omega\right)$, discretized with respect to the angle $\varphi \in[0,2 \pi]$. Specifically, let $\xi_{\boldsymbol{\rho}_{e}}$ represent an $\sqrt{m / 2}$ dimensional complex column vector, corresponding to $\hat{g}_{w}\left(\varphi, \boldsymbol{\rho}_{e} ; \omega\right)$ uniformly sampled at $\sqrt{m / 2}$ angular points $\varphi$. The vector $\xi_{\rho_{e}}$ represents the weights of plane waves incident upon the target due to a source at $\boldsymbol{\rho}_{e}$, at an $\sqrt{m / 2}$-dimensional set of discrete plane-wave propagation directions (angles). Recall that it is assumed that $\sqrt{m / 2}$ is an integer.

\section{B. Radiation from Induced Source}

Fields incident upon a scatterer induce currents on the target surface (and/or within the target), and the scattered fields are radiated by this source, with radiation taking place in the presence of the background medium. Therefore, to consider the scattering problem, we first address radiation from a general source, radiating from within the vacuum pocket in Figure 3 (the target will reside within this region). Specifically, assume that $i\left(\boldsymbol{\rho}^{\prime}, \omega\right)$ represents a source at frequency $\omega$, residing within the pocket vacuum region, and therefore $i\left(\left|\boldsymbol{\rho}^{\prime}\right| \geq \rho_{t}, \omega\right)=0$. Let $g\left(\boldsymbol{\rho}_{o}, \boldsymbol{\rho}^{\prime} ; \omega\right)$ represent the Green's function for a line source located at $\rho^{\prime}$, for $\left|\rho^{\prime}\right| \leq \rho_{t}$, and the associated fields are observed at any point $\boldsymbol{\rho}_{o}$; the Green's function takes into account the properties of the heterogeneities. The fields at $\boldsymbol{\rho}_{o}$ due to the source $i\left(\boldsymbol{\rho}^{\prime}, \omega\right)$, radiating in the presence of the heterogeneities, may therefore be expressed as

$$
f\left(\boldsymbol{\rho}_{o}, \omega\right)=\int d \boldsymbol{\rho}^{\prime 2} i\left(\boldsymbol{\rho}^{\prime}, \omega\right) g\left(\boldsymbol{\rho}_{o}, \boldsymbol{\rho}^{\prime} ; \omega\right)
$$

Because of the finite support of the current, we recall the window function $w\left(\boldsymbol{\rho}, \rho_{t}, \rho_{h}\right)$ introduced above, and window the Green's function as $g_{w}\left(\boldsymbol{\rho}_{o}, \boldsymbol{\rho}^{\prime} ; \omega\right)=g\left(\boldsymbol{\rho}_{o}, \boldsymbol{\rho}^{\prime} ; \omega\right) w\left(\boldsymbol{\rho}^{\prime}, \rho_{t}, \rho_{h}\right)$. Using Parseval's theorem, (3) may be expressed as 


$$
f\left(\boldsymbol{\rho}_{o}, \omega\right)=\frac{k_{o}}{4 \pi^{2}} \int_{0}^{2 \pi} d \varphi \hat{i}(\varphi, \omega) \hat{g}_{w}\left(\boldsymbol{\rho}_{o}, \varphi+\pi ; \omega\right)
$$

where $\hat{i}(\varphi, \omega)$ and $\hat{g}_{w}\left(\boldsymbol{\rho}_{o}, \varphi ; \omega\right)$ are spectral forms of the source current and windowed Green's function, respectively, defined similarly to (1), and in (4) it is assumed that the evanescent components of the spectral currents do not contribute to the fields observed at position $\rho_{o}$ (therefore the spectral current is a circle of radius $k_{o}$ in wavenumber space [17], and the general two-dimensional inverse spectral integral reduces to a one-dimensional integral around the aforementioned circle).

The more complicated the propagation medium between the observation point $\rho_{o}$ and the region in which the source resides, the more spectral angles $\varphi$ will contribute prominently to $\hat{g}_{w}\left(\boldsymbol{\rho}_{o}, \varphi+\pi ; \omega\right)$. Therefore the complex propagation medium between the source and observation point serves the role of sampling multiple angles $\varphi$ of radiation from the spectral source $\hat{i}(\varphi, \omega)$. By contrast, when the medium is characterized by vacuum, the spectral Green's function $\hat{g}_{w}\left(\boldsymbol{\rho}_{o}, \varphi+\pi ; \omega\right)$ is strongly peaked for $\varphi$ in the linear direction from the source to $\boldsymbol{\rho}_{o}$, and therefore in that case $f\left(\boldsymbol{\rho}_{o}, \omega\right)$ only samples spectral content of $\hat{i}(\varphi, \omega)$ narrowly centered about that direction.

\section{Scattering from Target Embedded in Heterogeneity}

Let $u\left(\phi_{i}, \varphi, \omega\right)$ represent the two-dimensional Fourier transform of the current induced on the target at spectral angle $\varphi$, due to a unit-amplitude plane-wave incident in vacuum at angle $\phi_{i}$ and frequency $\omega$ (recall that the discretized/vector version, discretized with respect to $\varphi$, is represented as $\tilde{u}$ in complex form and as $u$ as a corresponding real vector). If we ignore multiple interactions between the target and the background heterogeneities in Figure 3 (this is termed the Born approximation [18], and it is often appropriate for heterogeneities sufficiently distant from the target), then the Fourier transform of the induced currents on the target, due to the excitation $g_{w}\left(\boldsymbol{\rho}, \boldsymbol{\rho}_{e} ; \omega\right)$ discussed in Section II-A, may be expressed as

$$
\hat{i}(\varphi, \omega) \approx \int_{0}^{2 \pi} d \phi_{i} u\left(\phi_{i}, \varphi, \omega\right) \hat{g}_{w}\left(\phi_{i}, \boldsymbol{\rho}_{e} ; \omega\right)
$$

where we recall that $\hat{g}_{w}\left(\phi_{i}, \boldsymbol{\rho}_{e} ; \omega\right)$ represents the amplitude of plane-wave component propagating at angle $\phi_{i}$ within the overall excitation field $g_{w}\left(\boldsymbol{\rho}, \boldsymbol{\rho}_{e} ; \omega\right)$. In the subsequent analysis we assume 
the approximation in (5) to be accurate (thereby assuming that the Born approximation is accurate), with this issue revisited in Section II-E.

Let the $\sqrt{m / 2} \times \sqrt{m / 2}$ matrix $\tilde{U}$ represent a complex sampled version of $u\left(\phi_{i}, \phi_{s}, \omega\right)$, with the columns representing $\sqrt{m / 2}$ samples with respect to $\phi_{i} \in[0,2 \pi]$, and the rows representing $\sqrt{m / 2}$ samples with respect to $\phi_{s} \in[0,2 \pi]$ (when "unwrapped" $\tilde{U}$ represents the vector $\tilde{u}$ ). From (5) a discretized form of the induced current (at $\sqrt{m / 2}$ discrete $\varphi=\phi_{s}$ ) may be expressed as

$$
\hat{i} \approx \Delta_{\phi_{i}} \xi_{\rho_{e}}^{T} \tilde{U}
$$

where now $\hat{i}$ is in the form of an $\sqrt{m / 2}$-dimensional complex row vector, and $\Delta_{\phi_{i}}$ represents the sample rate of the angle $\phi_{i} \in[0,2 \pi]$. Equation (6) represents the induced target current in the spectral domain as a weighted sum of spectral currents, with the weighting (complex vector) $\xi_{\boldsymbol{\rho}_{e}}$ defined by the amplitude of $\sqrt{m / 2}$ plane waves propagating at discrete angles $\phi_{i} \in[0,2 \pi]$.

Using (4), the fields scattered from the target situated within the presence of the heterogeneity, as observed at $\boldsymbol{\rho}_{o}$, is given approximately as

$$
v\left(\boldsymbol{\rho}_{o}, \boldsymbol{\rho}_{e}, \omega\right) \approx \frac{k_{o} \Delta_{\phi_{i}} \Delta_{\phi_{s}}}{4 \pi^{2}} \xi_{\boldsymbol{\rho}_{e}}^{T} \tilde{U} \zeta_{\boldsymbol{\rho}_{o}}
$$

where $\zeta_{\boldsymbol{\rho}_{o}}$ represents $\xi_{\boldsymbol{\rho}_{o}}$ with a shift of the angle $\varphi$ by $\pi$, commensurate with (4); $v\left(\boldsymbol{\rho}_{o}, \boldsymbol{\rho}_{e}, \omega\right)$ is a complex number corresponding to a measurement for a source at $\boldsymbol{\rho}_{e}$ and observer at $\boldsymbol{\rho}_{o}$. A total of $n / 2$ such measurements (for $n / 2$ combinations of the pair $\left(\boldsymbol{\rho}_{e}, \boldsymbol{\rho}_{o}\right)$ ) yields an $n$-dimensional real CS vector $v$, corresponding to the concatenated real and imaginary parts of these $n / 2$ measurements. The approximation in (7) is manifested by discretization with the angles $\phi_{i}$ and $\phi_{s}$, and it is exact in the limit $\Delta_{\phi_{i}} \rightarrow 0$ and $\Delta_{\phi_{s}} \rightarrow 0$.

As elucidated further below, (7) corresponds to a compressive-sensing measurement performed at observation point $\boldsymbol{\rho}_{o}$ due to a line source at $\boldsymbol{\rho}_{e}$; importantly, note that the measured data $v\left(\boldsymbol{\rho}_{o}, \boldsymbol{\rho}_{e}, \omega\right)$ is a linear combination of the discretized components of $u\left(\phi_{i}, \phi_{s}, \omega\right)$, represented by the matrix $\tilde{U}$, with the linear weights driven by the discretized Green's functions $\xi_{\rho_{e}}$ and $\zeta_{\boldsymbol{\rho}_{o}}$; these weights therefore capture the complexity of the propagation medium, with the number of significant spectral angles in $\xi_{\boldsymbol{\rho}_{e}}$ and $\zeta_{\boldsymbol{\rho}_{o}}$ increasing with increasing propagation complexity. The vector $\xi_{\boldsymbol{\rho}_{e}}$ defines the diversity of plane waves contributing to exciting the target, and $\zeta_{\boldsymbol{\rho}_{o}}$ defines the diversity of scattering angles from the target contributing to the measurement at $\boldsymbol{\rho}_{o}$. 


\section{Compressive Sensing Perspective}

The matrix $\tilde{U}$ defines the induced target spectral current $u\left(\phi_{i}, \phi_{s}\right)$ at $\sqrt{m / 2} \times \sqrt{m / 2}$ discrete sets of angles $\left(\phi_{i}, \phi_{s}\right)$, and rather than viewing it as a matrix, it may be "unwrapped" to constitute an $m / 2$-dimensional column vector $\tilde{u}$. The $m$-dimensional real vector $u$ corresponds to $\tilde{u}$ with the real and imaginary parts concatenated. Further, the linear relationship between the (single) compressive measurement $v\left(\boldsymbol{\rho}_{o}, \boldsymbol{\rho}_{e}, \omega\right)$ and $u$, as defined by (7), may be expressed in terms of the $m \times 2$ dimensional matrix $\sigma_{\boldsymbol{\rho}_{o}, \boldsymbol{\rho}_{e}}$, and therefore

$$
v_{\boldsymbol{\rho}_{o}, \boldsymbol{\rho}_{e}}=\sigma_{\boldsymbol{\rho}_{o}, \boldsymbol{\rho}_{e}}^{T} u
$$

with $v_{\boldsymbol{\rho}_{o}, \boldsymbol{\rho}_{e}}$ a two-dimensional vector corresponding to the real and imaginary parts of $\tilde{v}\left(\boldsymbol{\rho}_{o}, \boldsymbol{\rho}_{e}, \omega\right)$

Assume $n / 2$ such compressive-sensing measurements are performed, at $n / 2$ different pairs of source-observation positions $\left(\boldsymbol{\rho}_{o}, \boldsymbol{\rho}_{e}\right)$, then the compressive-sensing measurements at frequency $\omega$ may be expressed in terms of an $n$-dimensional real column vector $v$, and

$$
v=\Sigma u
$$

where two consecutive rows of $\Sigma$ correspond to terms of the form $\sigma_{\boldsymbol{\rho}_{o}, \boldsymbol{\rho}_{e}}^{T}$. The weights on the CS measurements, driven by the matrix $\Sigma$ are dictated by the complexity of the propagation medium. In this sense, the more complicated the medium the better, as in time reversal [14][16]. As discussed by Donoho [7], a wide range of $\Sigma$ (and hence projections of $u$ ) should be appropriate for compressive sensing.

All discussions above have assumed operation at a single frequency $\omega$. The discussion may be extended to multiple frequencies. Because of the assumed linear scattering, the different frequencies may be characterized independently, and therefore the matrix $\Sigma$ generalizes to a larger matrix; this matrix is block diagonal with each block corresponding to a particular frequency and of the form discussed above. In this context, we may similarly generalize the single-frequency vectors $v$ and $u$ by concatenating such vectors associated with different frequencies.

For simplicity in the discussion that follows we will continue to assume operation at a single frequency. Typically the number of compressive sensing measurements $n \ll m$, and therefore direct inversion for the desired $u$ based on the measured data $v$ is not possible. However, the 
inversion may be performed by exploiting properties of the parameters in $u$. Specifically, the function $u\left(\phi_{i}, \phi_{s}, \omega\right)$ is generally a smooth function of variables $\left(\phi_{i}, \phi_{s}, \omega\right)$, which implies that a wavelet (or other appropriate orthonormal) transform of the discretized $u\left(\phi_{i}, \phi_{s}, \omega\right)$ should be sparse, implying that the associated wavelet coefficients should have a large fraction of negligibly small coefficients.

Letting $\theta$ represent the vector of $m$ wavelet coefficients (or other orthonormal transform in which $u$ is sparse), and letting $\Psi$ represent the $m \times m$ wavelet-basis matrix, then $u=\Psi \theta$ and

$$
v=\Sigma \Psi \theta=\Phi \theta
$$

There have been several techniques developed in compressive-sensing theory [6], [8]-[13] for inversion of $\theta$ based on the observed $v$, typically imposing an $\ell_{1}$ regularization on $\theta$. The specific technique considered here is discussed in Section III, when presenting results.

\section{E. Revisiting Approximations}

In Section II-A we commenced by stating the goal of recovering $u_{s}\left(\phi_{i}, \phi_{s}, \rho_{s}, \omega\right)$ for a large range of $\left(\phi_{i}, \phi_{s}, \rho_{s}, \omega\right)$, where $u_{s}\left(\phi_{i}, \phi_{s}, \rho_{s}, \omega\right)$ represents the complex scattered field of a target situated in vacuum, as viewed in the far-zone at $\left(\phi_{s}, \rho_{s}\right)$, due to plane-wave excitation at angle $\phi_{i}$ and frequency $\omega$. The compressive-sensing analysis above sought to recover $u\left(\phi_{i}, \phi_{s}, \omega\right)$, which represents the Fourier transform of the associated induced currents, at spectral angle $\phi_{s}$. The desired $u_{s}\left(\phi_{i}, \phi_{s}, \rho_{s}, \omega\right)$ may be recovered from $u\left(\phi_{i}, \phi_{s}, \omega\right)$ via the free-space Green's function, and in the far-zone

$$
u_{s}\left(\phi_{i}, \phi_{s}, \rho_{s}, \omega\right)=\frac{\exp \left(-j k_{o} \rho_{s}-j \pi / 4\right)}{4 \pi} \sqrt{\frac{2 \pi}{k_{o} \rho_{s}}} u\left(\phi_{i}, \phi_{s}, \omega\right)
$$

The desire for $u_{s}\left(\phi_{i}, \phi_{s}, \rho_{s}, \omega\right)$ motivated assuming the Born approximation in (5). However, if the Born approximation is not valid, this does not undermine the overarching compressivesensing analysis. It simply implies that the associated scattered fields in (11) also includes effects of the induced current introduced by interaction with the heterogeneous background. Hence, rather than recovering the induced current of the target due to scattering within a vacuum, the effects of the heterogeneity are also included (although, as indicated, these are often weak). In such an analysis one still exploits the spectral diversity characterized by $\xi_{\boldsymbol{\rho}_{e}}$ and $\zeta_{\boldsymbol{\rho}_{o}}$, and this 
diversity may be of importance in classifying such a target embedded in a complex propagation environment (with classification based on the recovered $u\left(\phi_{i}, \phi_{s}, \omega\right)$, which is an approximation to the induced currents on the target when situated in free space).

\section{APPLICATIONS}

\section{A. Target Between Perfectly-Reflecting Plates}

As a first application of the theory introduced in Section II, we consider a target situated between two infinitely long and wide perfectly-reflecting plates. As is well known, a line source between these plates, required for computation of the Green's functions $\xi_{\boldsymbol{\rho}_{e}}$ and $\zeta_{\boldsymbol{\rho}_{o}}$, may be reconstituted in terms of an infinite number of such sources residing in vacuum (using image theory [19]); recall that $\xi_{\boldsymbol{\rho}_{e}}$ and $\zeta_{\boldsymbol{\rho}_{o}}$ correspond to the Green's function in the spectral domain. To constitute the spectral-domain Green's function of a source between the two plates, we first consider the spectral form of the Green's function of a line source in vacuum. Specifically, it is well known that the far-zone Green's function for a line source of unit amplitude may be expressed as [17]

$$
g\left(\boldsymbol{\rho}, \boldsymbol{\rho}^{\prime} ; \omega\right) \approx \frac{k_{o}}{4 \pi} \int_{0}^{2 \pi} d \varphi \cos (\varphi) \exp \left[-j k_{o}\left\|\boldsymbol{\rho}-\boldsymbol{\rho}^{\prime}\right\| \cos (\varphi-\phi)\right]
$$

where $\rho$ locates the observation point, $\rho^{\prime}$ locates the source point, and $\phi$ represents the corresponding angle between the two; real spectral angles $\varphi$ are considered because the evanescent fields are here ignored, under the far-zone assumption [17]. Therefore, each line source generates a continuum of plane waves that are incident upon the target, and each such plane wave corresponds to one spectral component with associated propagation angle $\varphi$. These spectral components are responsible for the spectral-domain Green's function $\hat{g}_{w}\left(\varphi, \rho_{e} ; \omega\right)$ from one image line current to the target phase center. Similarly, from the phase center of the target to a particular image observation point, there is a continuum of spectral plane waves, these constituting $\hat{g}_{w}\left(\boldsymbol{\rho}_{o}, \varphi ; \omega\right)$.

If the distance $\left\|\rho-\rho^{\prime}\right\|$ is several wavelengths or more, there is an associated stationary point in the direction linking the source image and the target center (for the excitation images), with a similar phenomenon manifested for the scattered fields; the integral of continuous spectral plane waves reduces to a dominant space-domain plane wave propagating in the direction to or 
from the source/observer and the target phase center (i.e., one spectral component dominates). Specifically, the integral in (12) may expressed approximately as

$$
g\left(\boldsymbol{\rho}, \boldsymbol{\rho}^{\prime} ; \omega\right) \approx \frac{\exp \left(-j k_{o}\left\|\boldsymbol{\rho}-\boldsymbol{\rho}^{\prime}\right\|-j \pi / 4\right)}{4 \pi} \sqrt{\frac{2 \pi}{k_{o}\left\|\boldsymbol{\rho}-\boldsymbol{\rho}^{\prime}\right\|}}
$$

Therefore, within the constitution of the $\operatorname{CS}$ matrix $\Phi$, the contribution from a given image source at $\rho^{\prime}$ is represented by a single weighted angle-dependent term of the form in (13); i.e., the vectors $\xi_{\boldsymbol{\rho}_{e}}$ and $\zeta_{\boldsymbol{\rho}_{o}}$ associated with a given image term are defined by a single non-zero angular component, corresponding to the associated angle between $\boldsymbol{\rho}^{\prime}$ and $\boldsymbol{\rho}$.

Returning to (7), the fields due to an excitation within the waveguide at $\boldsymbol{\rho}_{e}$, as observed within the waveguide at $\boldsymbol{\rho}_{o}$, with the target phase center at the origin $\boldsymbol{\rho}=0$, may be expressed as

$$
v\left(\boldsymbol{\rho}_{o}, \boldsymbol{\rho}_{e}, \omega\right) \approx \frac{k_{o} \Delta \phi_{i} \Delta \phi_{s}}{4 \pi^{2}} \xi_{\boldsymbol{\rho}_{e}}^{T} \tilde{U} \zeta_{\boldsymbol{\rho}_{o}}
$$

and we sum (14) across the contributions from all images (each with its own pair of $\xi_{\rho_{e}}$ and $\left.\zeta_{\rho_{o}}\right)$.

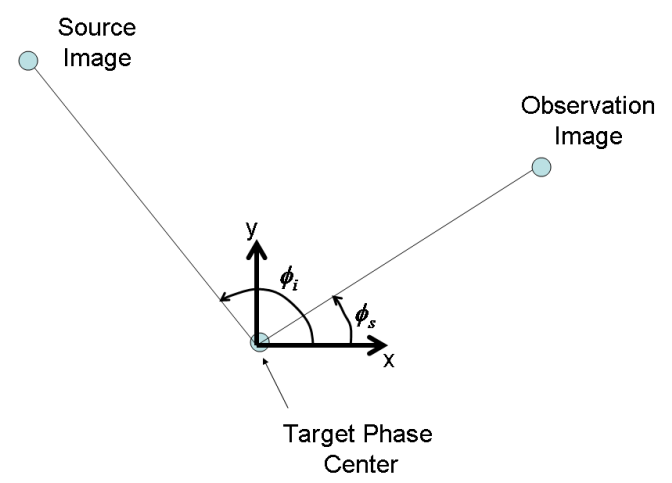

Fig. 6. Coordinates and angles of source and receiver images. Only two such images are shown, although there are an infinite number of images.

The scattering problem considered is depicted in Figure 7, wherein properties of the finite difference time-domain (FDTD) [20] analysis method are represented, including placement of perfectly matched layer (PML) [21] regions. The time-domain ring down of this target takes a significant amount of time, and this yields numerical artifacts in the computed data due to 
associated numerical dispersion [20]. In addition, in the CS computations we must consider numerous different source positions, and each require a long FDTD run to capture the entire time-domain ring down. Therefore, the data used in the CS analysis was generated as follows. The dielectric target was first analyzed in free space using FDTD, with plane-wave excitation, at 256 angles over $0^{\circ} \leq \phi_{i} \leq 180^{\circ}$, and the scattered fields were observed at 256 angles over $0^{\circ} \leq \phi_{s} \leq 180^{\circ}$. After taking a Fourier transform of these (free-space target) time domain fields, which stop ringing far more quickly than those associated with Figure 7, the scattered fields are determined at the wavelength $\lambda$ associated with Figure 7. In the free-space FDTD analysis these scattered fields are computed a distance $15 \lambda$ from the target center, and the computational domain is surrounded by a PML. Using the relationship in (11), we estimate the Fourier transform of the induced current $u\left(\phi_{i}, \phi_{s}, \omega\right)$, at frequency $\omega$ corresponding to the wavelength $\lambda$ of interest. Finally, $u\left(\phi_{i}, \phi_{s}, \omega\right)$ is used with image theory (Green's function inside the two-plate region) to constitute the scattered fields. Since the different source positions are address efficiently within the image-theory analysis, consideration of many different source positions does not required a large amount of computation time.

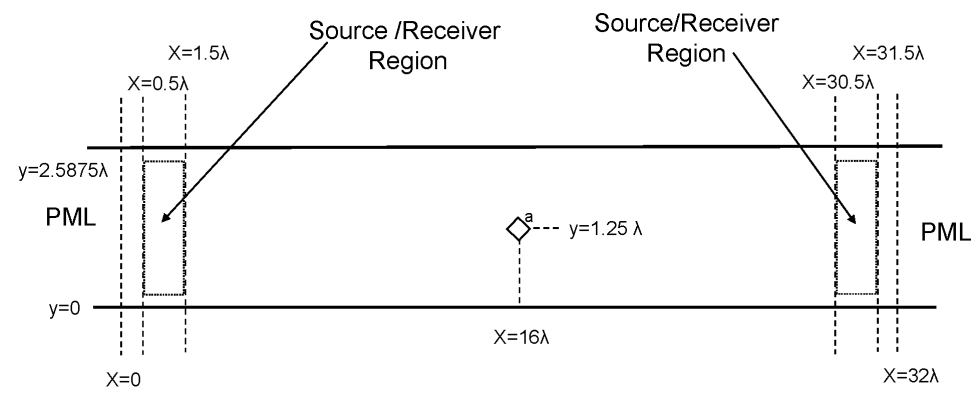

Fig. 7. Two-dimensional dielectric cylinder with square cross section situated between two perfectly-conducting plates. The scattered fields are computed via the finite difference time-domain (FDTD) method, and perfectly match layer (PML) regions are placed at each end. Although time-domain scattering computations are obtained, in the example considered compressive sensing (CS) is performed at a single frequency, and the dimensions are defined with respect to the corresponding wavelength $\lambda$. The CS line sources and line receivers are situated randomly within the corresponding regions depicted in the figure. The target is in the center, with square side width $a$, where $a=1 \lambda$ and the target is a lossless dielectric with dielectric constant $\epsilon_{r}=2$.

While the FDTD computations associated with Figure 7 are expected to deteriorate with increasing time, due to numerical dispersion, good results are anticipated at early times. To examine the accuracy of the simplified model discussed above (which integrates the free-space 
FDTD solution with an image theory analysis), in Figure 8 we present a (small) part of the time-domain signal scattered from the target in Figure 7; a Fourier transform is performed with respect to the image-based frequency-domain scattered fields, to yield the time-domain data for that model (the FDTD results are performed directly in the time domain). One observes from Figure 8 that at early times the agreement between the direct FDTD solution and the hybrid solution is generally good, with the main differences manifested in the strengths of the peaks. There are at least two reasons for the discrepancies observed in Figure 8: (i) numerical dispersion associated with the FDTD solution in Figure 7; and (ii) it was assumed that the freespace FDTD computations of the target used to estimate $u\left(\phi_{i}, \phi_{s}, \omega\right)$ (using the image-based Green's function) were in the far zone of the target, but the employed distance $(15 \lambda)$ from the target center may not have been sufficient (this distance was limited in the associated FDTD computations, with the goal of minimizing numerical dispersion). It is felt that the numerical data based on the FDTD-(image theory) hybrid is sufficient to examine the effectiveness of CS in recovering $u\left(\phi_{i}, \phi_{s}, \omega\right)$ based on a relatively small set of CS measurements. In practice, it is anticipated that this framework (target placed inside plates) will be employed to constitute a CS-based scattering measurement facility, and therefore the numerical issues associated with generating the test data will in that case be a non-issue.

Considering now CS reconstruction of $u\left(\phi_{i}, \phi_{s}, \omega\right)$, we consider a finite set of randomly chosen source and receiver positions within the source/receiver positions in Figure 7; in the examples considered, all transmitter and receiver positions are placed in the left region in Figure 7, and therefore the results correspond to $0^{\circ} \leq \phi_{i} \leq 180^{\circ}$ and $0^{\circ} \leq \phi_{s} \leq 180^{\circ}$. It is important to note that we observed better CS reconstruction performance when the source/receiver positions were selected randomly (within the associated regions in Figure 7), vis-à-vis placing the positions on a uniformly sampled grid within these regions. The basis $\Psi$ used in the CS analysis corresponds to a discrete cosine transform (DCT), for reasons discussed further below.

The reference real and imaginary part of $u_{s}\left(\phi_{i}, \phi_{s}, \rho_{s}, \omega\right)$ are depicted in Figure 9, where the discretization of this image corresponds to 256 bins in the angle $\phi_{i}$ and 256 bins in the angle $\phi_{s}$, corresponding to a real signal of size $m=2 \times 256 \times 256$; these scattered fields are normalized by $\exp \left(-j k_{o} \rho_{s}\right) / \sqrt{\rho_{s}}$. In Figure 10 are shown the real part of the CS reconstructions, with the imaginary parts depicted in Figure 11, based on 64 and 256 different placements of the transmitter-receiver pair (corresponding to $n=128$ and $n=512$ real CS measurements, 


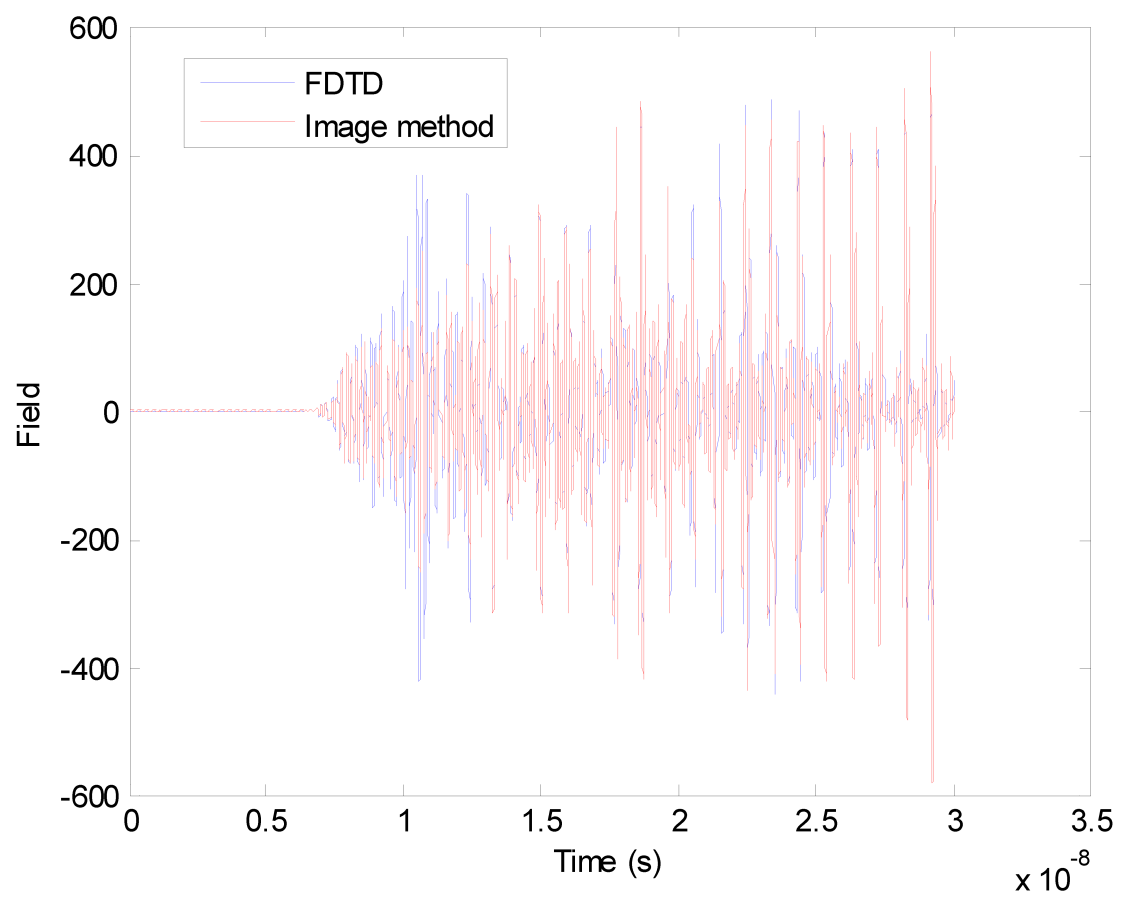

Fig. 8. Example comparison of time-domain fields computed for example source line source and receiver, with these elements placed on the left side of the domain (see Figure 7). One solution is computed using the FDTD analysis depicted in Figure 7, and the other (marked "Image") corresponds to an integration of the free-space target signature with image theory. Only a relatively short period of the entire time-domain signal is depicted.

respectively, accounting for the concatenated real and imaginary parts of the measured data). We observe that both 64 and 256 different random placements of the transmitters and receivers yields very good estimates for the normalized $u_{s}\left(\phi_{i}, \phi_{s}, \rho_{s}, \omega\right)$, which is proportional to $u\left(\phi_{i}, \phi_{s}, \omega\right)$. The CS inversion in these examples was performed using the algorithm described in [12].

It is of interest to discuss the utility of a setting like that in Figure 7 for performing multistatic scattering measurements. Consider conventional multi-static scattering measurement, with the transmitter and receiver antennas placed in the far zone, at respective angles $\phi_{i}$ and $\phi_{s}$, with absorbing material employed to mitigate scattering with the background. If one considers $0^{\circ} \leq \phi_{i} \leq 180^{\circ}$ and $0^{\circ} \leq \phi_{s} \leq 180^{\circ}$ with 256 angular samples in both $\phi_{i}$ and $\phi_{s}$, then a total of $256 \times 256=65,536$ measurements are required, this compared with the excellent CS results in Figures 10 and 11 based on only 64 measurements (a comparable number of measurements performed in a conventional setting would correspond to sampling $\phi_{i}$ and $\phi_{s}$ at an angular rate 

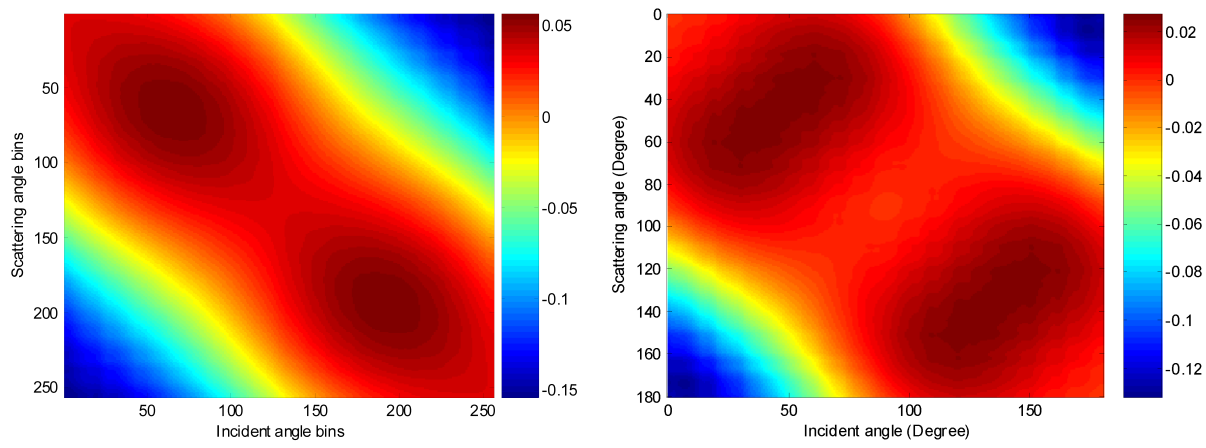

Fig. 9. Reference Fourier transform of the induced currents, $u\left(\phi_{i}, \phi_{s}, \rho_{s}, \omega\right)$, on the target in Figure 7. The reference solution was computed via an FDTD analysis, using plane-wave excitation with the target in vacuum. Left: real part of $u_{s}\left(\phi_{i}, \phi_{s}, \rho_{s}, \omega\right)$, right: imaginary part of $u_{s}\left(\phi_{i}, \phi_{s}, \rho_{s}, \omega\right)$. The scattered fields are normalized with respect to $\exp \left(-j k_{o} \rho_{s}\right) / \sqrt{\rho_{s}}$.

of approximately $22.5^{\circ}$, which is likely too coarse for usable data). At least as important to the number of measurements required is the convenience of the measurements. The framework in Figure 7 does not require absorbing material all around the domain (only absorbing material in the location of the PML employed in the numerical computations). For the far-zone approximation to be valid in Figure 7 the length of the waveguide need be sufficiently long, but the target may be placed relatively close to the plates. There is still much work to be done in optimizing such a CS-based setting for performing multi-static scattering measurements, but the results presented here suggest that this may be a fruitful direction of future research.
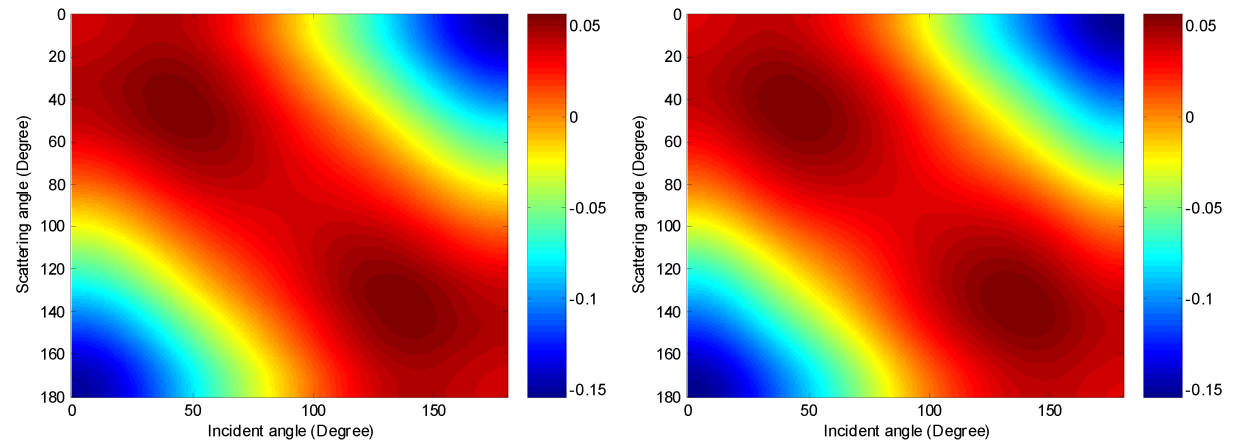

Fig. 10. Real part of the CS estimation of $u_{s}\left(\phi_{i}, \phi_{s}, \rho_{s}, \omega\right)$, based on 64 source-receiver positions (left) and 256 source-receiver positions (right). The scattered fields are normalized with respect to $\exp \left(-j k_{o} \rho_{s}\right) / \sqrt{\rho_{s}}$. 

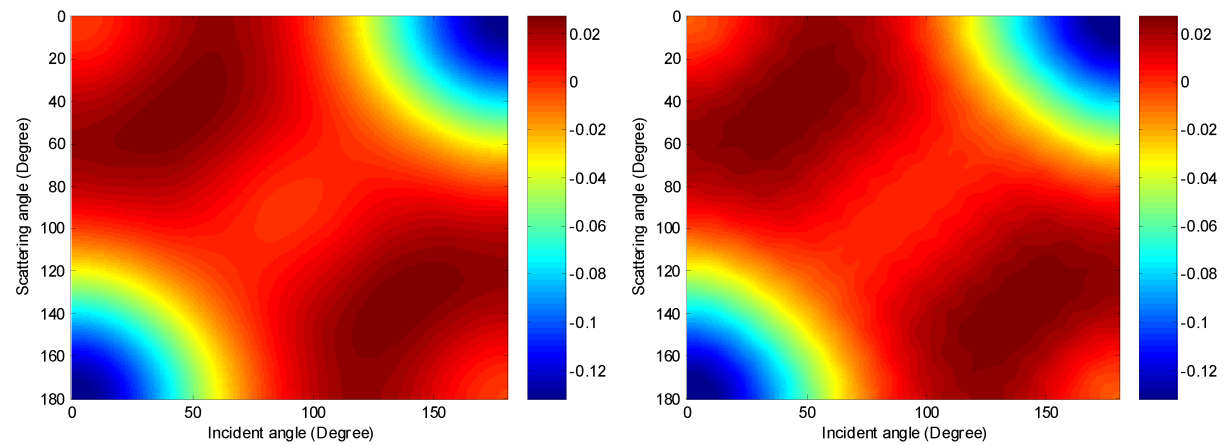

Fig. 11. Imaginary part of the CS estimation of $u_{s}\left(\phi_{i}, \phi_{s}, \rho_{s}, \omega\right)$, based on 64 source-receiver positions (left), and 256 source-receiver positions (right). The scattered fields are normalized with respect to $\exp \left(-j k_{o} \rho_{s}\right) / \sqrt{\rho_{s}}$.

\section{B. Accelerating Linear Scattering Computations}

The computational setting in Figure 7 was employed in lieu of a measurement facility, to demonstrate the idea of CS-based multi-static scattering measurements but, as indicated, it is felt that such a framework is most appropriate for simplifying scattering measurements. We now discuss how the CS ideas may be explicitly employed within the context of multi-static scattering computations, here with example to electromagnetic scattering.

Most linear scattering analyses result in a matrix equation of the form

$$
Z i=v
$$

where $i$ represents a column vector of basis-function coefficients to be solved for, and the column vector $v$ represents the known source, or excitation. If the analysis involves $N_{b}$ basis function coefficients, then $i$ and $v$ are complex vectors of dimension $N_{b}$, and $Z$ is an $N_{b} \times N_{b}$ complex matrix. An example numerical technique that yields matrix equations of the form in (15) is the method of moments [22]. When the dimensions of the target become large with respect to excitation wavelength (most such analyses are performed one frequency or wavelength at a time), then the number of basis functions $N_{b}$ may become quite large. Therefore, the order $N_{b}^{2}$ computational cost associated with filling $Z$, and the cost of performing the matrix inversion for $i$, become prohibitive. Once $i$ is computed for a particular excitation $v$, the scattered fields observed at different angles with respect to the target may be computed efficiently [22]. Therefore, the principal computational bottleneck is manifested in forming and solving (15) for a particular 
angle of incidence. This computational challenge has motivated development of techniques that allow (15) to be solved more efficiently than a naive direct solution, with the fast multipole method [23]-[25] constituting an important example. These latter techniques require $O\left(N_{b} \log N_{b}\right)$ computational cost to solve for $i$, and $O\left(N_{b}\right)$ computational cost to solve for the associated scattered fields after $i$ is obtained. Even with these fast techniques, forming and solving (15) for the unknown $i$ becomes computationally prohibitive as the size of the target increases relative to wavelength, i.e. with increasing $N_{b}$.

The analysis in (15) may be viewed as a computational sensing experiment: given the excitation $v$, the goal is to perform a computational "experiment" to estimate $i$, and from $i$ the scattered field. Hence, the same ideas from compressive sensing, which have typically been applied to physical experiments, may also be applied to computational experiments. One may therefore ask the following question: Is it possible to employ CS to reduce the number of times we must solve (15), and, based upon this reduced set of calculations, can the full multi-static scattering profile be recovered? In this context the "sensing" part of CS corresponds to executing a numerical computation, and the objective is to employ CS to reduce the number of required computations.

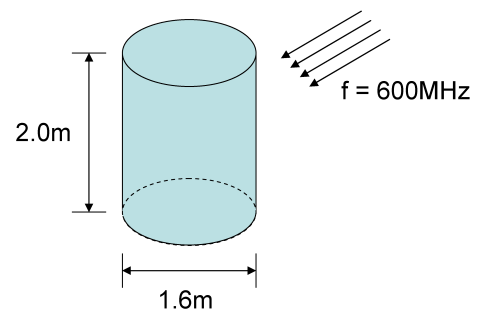

Fig. 12. Finite dielectric cylindrical target considered in three-dimensional fast-multipole electromagnetic scattering calculation. The target is a lossless dielectric with dielectric constant $\epsilon_{r}=2$.

We here examine this question within the context of electromagnetic scattering from the target in Figure 12. Scattering from this target was analyzed using the multi-level fast-multipole method (MLFMM) technique discussed in [25]. To simplify the analysis, the calculations were performed at a fixed azimuthal angle $\phi_{i}=\phi_{s}=0^{\circ}$, and the incidence and scattering angles were varied from $0^{\circ} \leq \theta_{i} \leq 180^{\circ}$ and $0^{\circ} \leq \theta_{s} \leq 180^{\circ}$. The MLFMM code was run with plane-wave incidence to sample $\theta_{i} 128$ times, and the far-zone scattered fields were computed at 128 angles $\theta_{s}$; these scattered fields provide a reference solution for the CS-based computations. The real 
and imaginary parts of the normalized scattered fields are depicted in Figure 14, where the fields are normalized with respect to $\exp \left(-j k_{o} r\right) / r$, where $r$ is the range from the target center to the (distant) receiver.

As a brief aside, we provide justification now for choosing the DCT as the basis $\Psi$ in the CS analysis presented here. In Figure 13 we compare the sparseness with which the data in Figure 12 are rendered for different $\Psi$. In Figure 13 are shown the ordered amplitudes when $\Psi$ corresponds to a discrete Fourier transform, a Harr wavelet [1], and a DCT. For the scattering data considered, the DCT provides the sparsest representation, and therefore this has been selected for all CS results presented here. However, as discussed in the Conclusions, further research is of interest concerning the selection of $\Psi$ for scattering computations and measurements.

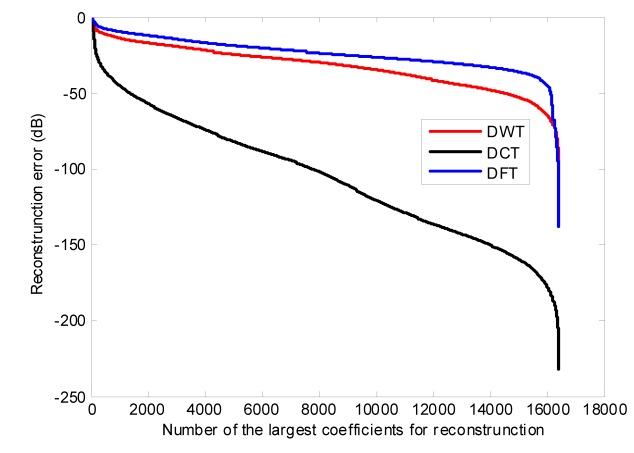

Fig. 13. Comparison of the ordered basis-function weights (magnitude) for the scattering data considered in Figure 14 , for $\Psi$ defined by a discrete Fourier, discrete cosine and Harr wavelet basis.

The MLFMM code was now run in a CS mode. Specifically, rather than assuming plane-wave excitation, the excitation $v$ in (15) was composed of a linear combination of plane waves, with the weights on this superposition drawn iid from a Gaussian random variable, with zero mean and unit variance. To design the excitation, the plane-wave incidence angle $\theta_{i}$ was sampled uniformly 128 times over $0^{\circ} \leq \theta_{i} \leq 180^{\circ}$, and each of the 128 plane waves was weighted by a unique draw from the aforementioned random variable, and then all plane waves were superposed to constitute one realization of $v$; importantly, using a $v$ so constituted requires no more cost to solve (15) than if $v$ corresponds to a plane wave. Once $i$ is computed for a given $v$, the scattered fields are computed in a CS mode, corresponding to a weighted sum of the scattered fields at 128 uniformly spaced $\theta_{s}$ over the range $0^{\circ} \leq \theta_{s} \leq 180^{\circ}$, with the weights again 
iid draws from the same random variable. Within the computational electromagnetic scattering engine, this weighted sum of fields from 128 scattering angles are computed at once, with no more computational cost than observing the scattered fields at a single scattering angle $\theta_{s}$. This process yields a single CS measurement. Let $N_{e}$ represent the number of different randomly constituted excitations $v$ considered (defined by different random-variable draws), and for each we consider $N_{s}$ random projections as a function of scattering angle, for a total of $N_{e} \times N_{s}$ CS computations. Since, as discussed above, the solution in (15) for $i$ is the computational bottleneck (at best $O\left(N_{b} \log N_{b}\right)$ ), the CS computations will be performed with the goal of minimizing $N_{e}$, recognizing that there is negligible additional cost associated with increasing $N_{s}$ (recall that after $i$ is computed, the scattered fields are computed with $O\left(N_{b}\right)$ complexity). The goal is to use these $N_{e} \times N_{s}$ computations to recover an estimate of $u_{s}\left(\phi_{i}, \phi_{s}, r, \omega\right)$ via CS analysis (here again using the CS-inversion algorithm described in [12]).
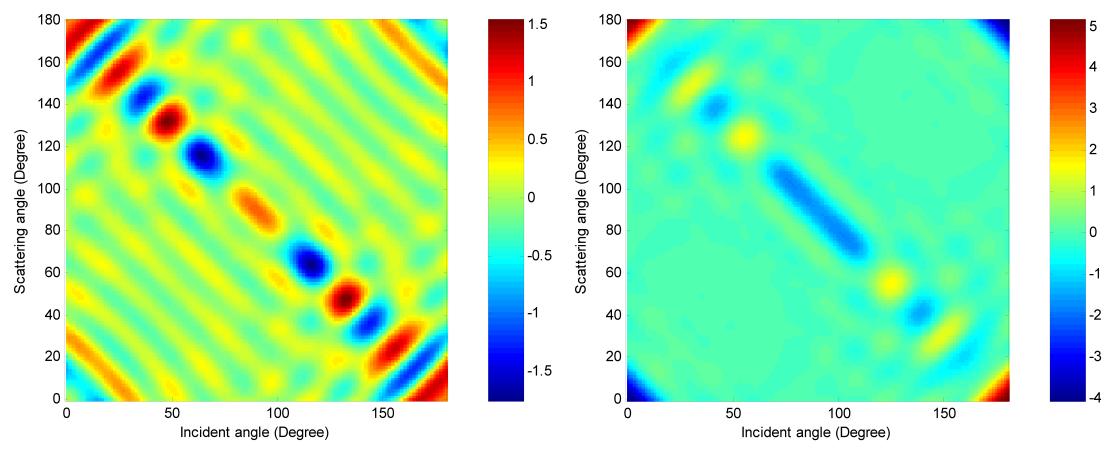

Fig. 14. Real (left) and imaginary (right) parts of the normalized scattered fields, as computed via a reference MLFMM [25] computation, with plane-wave excitation and the scattered fields observed in the far zone, as a function of scattering angle $\theta_{s}$. The scattered fields are normalized with respect to $\exp \left(-j k_{o} r\right) / r$.

In Figures 15 and 16 are shown respectively the CS-reconstructed real and imaginary parts of the scattered fields, for which one may compare to the reference solution in Figure 14. In these computations the number of randomly constituted excitations was fixed at $N_{e}=16$, and for each excitation the number of different random projections of the scattered fields was $N_{s}=16$, $N_{s}=32$ and $N_{s}=64$. The number of complex basis function coefficients was $N_{b}=20016$ (this is a relatively small number of unknowns, selected for efficient computation of the reference solution in Figure 14). While the results for $N_{s}=8$ (not shown) are reasonably good, they are substantially worse than those in Figures 15 and 16. We observe from Figures 15 and 16 that 
the CS-based MLFMM computations and associated CS reconstructions are in good agreement with the "truth" in Figure 14 (a quantitative comparison is provided below).
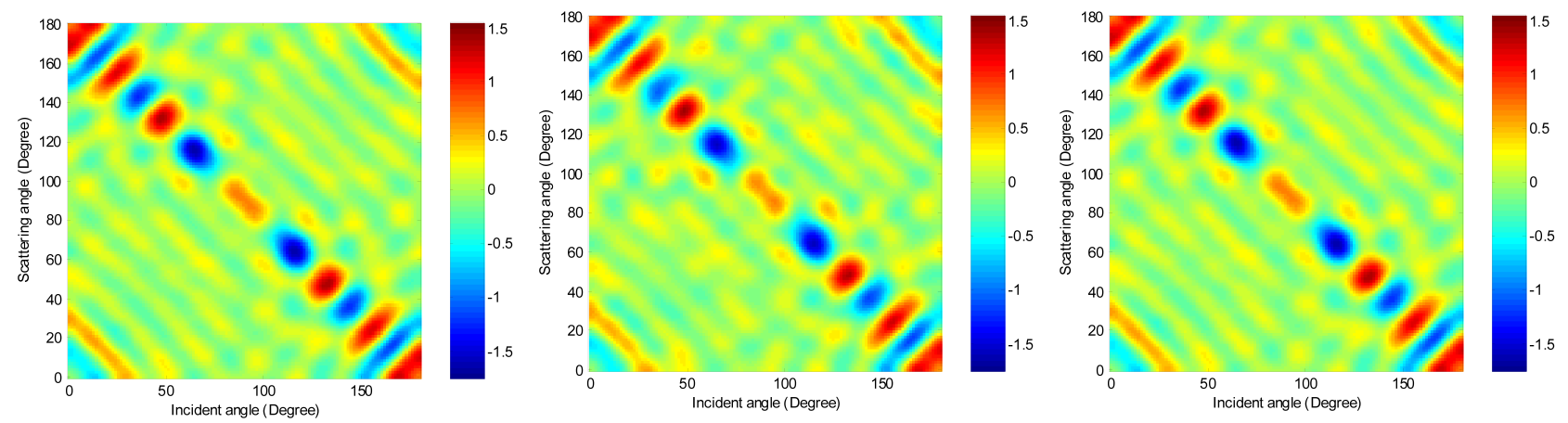

Fig. 15. Real part of the normalized scattered fields, as estimated by compressive sensing. The CS calculations were performed with $N_{e}=16$ random excitations, and the number of random CS scattering computations considered is $N_{s}=16$ (left), $N_{s}=32$ (center) and $N_{s}=64$ (right). The scattered fields are normalized with respect to $\exp \left(-j k_{o} r\right) / r$.
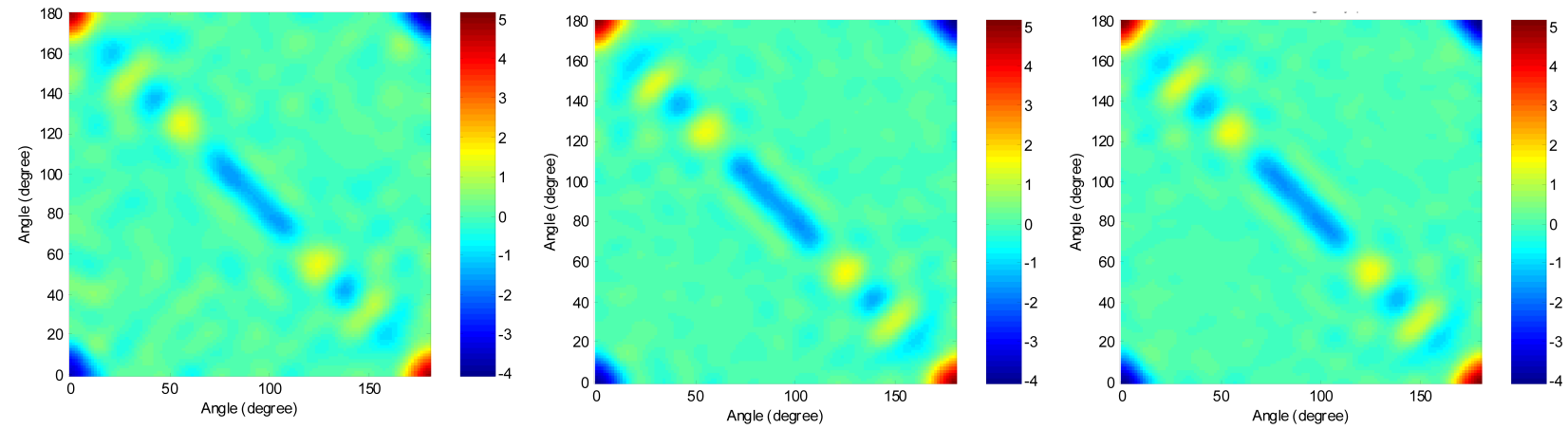

Fig. 16. Imaginary part of the normalized scattered fields, as estimated by compressive sensing. The CS calculations were performed with $N_{e}=16$ random excitations, and the number of random CS scattering computations considered is $N_{s}=16$ (left), $N_{s}=32$ (center) and $N_{s}=64$ (right). The scattered fields are normalized with respect to $\exp \left(-j k_{o} r\right) / r$.

To provide a comparison of the accuracy of the CS reconstructions, in Figure 17 is shown the real part of the normalized scattered field, for plane-wave excitation at $N_{e}=16$ angles over $0^{\circ} \leq \theta_{i} \leq 180^{\circ}$, and with the scattered field observed at $N_{s}$ different discrete angles (as in conventional scattering computations) over $0^{\circ} \leq \theta_{s} \leq 180^{\circ}$; as in the CS computations, we consider $N_{s}=16, N_{s}=32$ and $N_{s}=64$. The computational cost for this analysis is the same as that associated with the CS-based analysis in Figure 15. Although not shown here, for brevity, the imaginary component of these scattered fields, based on linear interpolation of the uniformly sampled results in $\theta_{e}$ and $\theta_{s}$, have a similar level of accuracy when compared to 
the CS-based results in Figure 16. The computation of each CS analysis, for a given random projection on excitation and on receive, is exactly of the same complexity as when plane-wave excitation is considered, and the scattered fields are observed at a single angle of observation (the CS inversion is performed in seconds in Matlab, and therefore this part of the analysis is a tiny fraction of the time required for the MLFMM scattering analysis).

To provide a quantitative measure of the difference between the CS-computed and traditionallycomputed (plane-wave excitation, and observation at discrete scattering angles), we consider the error $\left\|I_{\text {est }}-I_{\text {ref }}\right\|_{2} /\left\|I_{\text {ref }}\right\|_{2}$, where $I_{\text {est }}$ is the estimated $\left(\theta_{i}, \theta_{s}\right)$ dependent scattered field (complex) and $I_{r e f}$ is the associated reference solution depicted in Figure 14. The $I_{e s t}$ is estimated in two different ways, each requiring the same computational cost: (i) the CS-based approach with results reflected in Figures 15 and 16, and (ii) the "traditional" approach with uniform sampling in $\left(\theta_{i}, \theta_{s}\right)$ with results depicted (real part) in Figure 17. In Table I these errors are shown, for $N_{e}=16$ source excitations, and for $N_{s}=16, N_{s}=32$ and $N_{s}=64$. It is observed that the CS-based results are significantly more accurate than the corresponding uniform-sampled results. To realize accuracy commensurate with the CS solution, the uniformly sampled results require one to consider $N_{e}=32$ excitations, which more than doubles the computational cost.

In the traditional uniform-sampled results, linear interpolation was used to extrapolate to angles $\theta_{i}$ and $\theta_{s}$ not explicitly considered via the MLFMM analysis. Therefore, it is possible that the results of the uniform-sampled analysis may be improved by considering a more-sophisticated regression model (e.g., splines or a polynomial model). Therefore, the CS results considered here may be viewed from two perspectives: (i) as compared to simple linear interpolation on a uniformly sampled lattice in $\theta_{i}$ and $\theta_{s}$, the CS results yield improved accuracy with the same computational cost; and (ii) the CS analysis may be viewed as integrating the scattering computations with the regression, with the CS inversion providing a smooth and accurate representation of the scattered fields for a finely sampled lattice in $\theta_{i}$ and $\theta_{s}$.

\section{CONCLUSIONS}

Compressive sensing has been examined as a framework for efficiently performing scattering measurements or computations. Concerning measurements, it has been demonstrated that if the target is placed within the presence of a complex propagation medium, and the Green's function of this medium is known, then the multi-static fields scattered from this target may be measured in 

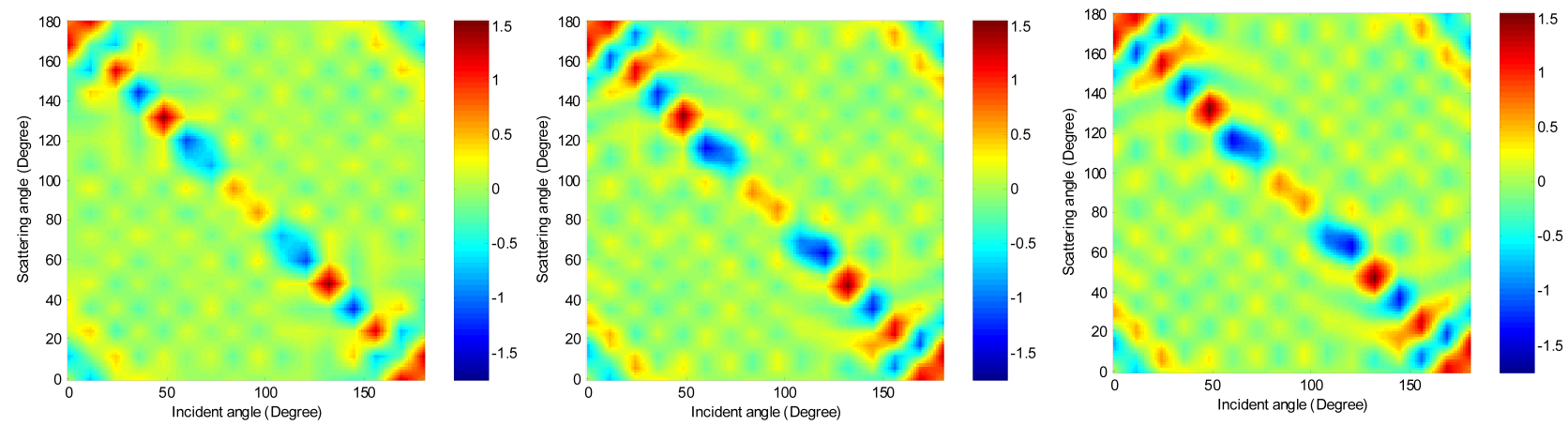

Fig. 17. Normalized scattered fields (real part) computed using random $N_{e}=16$ plane-wave incident angles uniformly sampled over the range $0^{\circ} \leq \theta_{i} \leq 180^{\circ}$, and the scattered fields are uniformly sampled at $N_{s}=16$ (left), $N_{s}=32$ (center) and $N_{s}=64$ (right) scattering angles, over $0^{\circ} \leq \theta_{i} \leq 180^{\circ}$. Linear interpolation is used to estimate the scattered fields at angles $\theta_{i}$ and $\theta_{s}$ for which the numerical scattered fields were not explicitly analyzed.

TABLE I

THE ERROR $\left\|I_{e s t}-I_{r e f}\right\|_{2} /\left\|I_{r e f}\right\|_{2}$, WHERE $I_{e s t}$ IS THE ESTIMATED $\left(\theta_{i}, \theta_{s}\right)$-DEPENDENT SCATTERED FIELD AND $I_{r e f}$ IS THE ASSOCIATED REFERENCE SOLUTION. THE $I_{e s t}$ IS ESTIMATED IN TWO WAYS: (I) THE CS-BASED APPROACH AND (II) THE “TRADITIONAL” UNIFORM ANGULAR SAMPLING. IN ALL CASES THE NUMBER OF EXCITATIONS IS $N_{e}=16$, AND THE RESULTS ARE PRESENTED FOR DIFFERENT NUMBERS OF SCATTERING COMPUTATIONS $N_{s}$. THE RESULTS FOR THE ERROR ON THE REAL AND IMAGINARY PART OF THE SCATTERED FIELDS ARE DENOTED “REAL,IMAGINARY”.

\begin{tabular}{|c|c|c|c|}
\hline & $N_{s}=16$ & $N_{s}=32$ & $N_{s}=64$ \\
\hline Uniform Sampling & $0.51,0.35$ & $0.38,0.25$ & $0.36,0.24$ \\
\hline Compressive Sensing & $0.38,0.30$ & $0.25,0.16$ & $0.14,0.12$ \\
\hline
\end{tabular}

a highly efficient manner using ideas from CS (this has been referred to as in situ CS, because the background medium itself, and the associated Green's function, is used to constitute the CS projections on the angle-dependent target scattering response). In particular, for a given resolution of the angle-dependent target scattered fields, the total number of CS scattering measurements may be significantly less than the number required if one were to performed free-space measurements. The more-complicated the background propagation medium, the more resolution is possible given a finite set of CS measurements (source/receiver positions). A practical application of this idea was demonstrated using a numerical experiment, wherein a highly scattering environment with known Green's function was constituted by placing a target within a medium bounded by planar perfectly conducting walls. In this setting the multi-aspect scattered fields were efficiently analyzed, with this providing a possible future alternative to 
anechoic-chamber-based measurement systems. The idea of exploiting a complex propagation medium to reduce the number of measurements required for characterization of a source or target has also been investigated in the related field of time reversal [14]-[16], to which connections have been made.

In the context of a numerical scattering analysis, one typically considers plane-wave excitation, with the scattered fields computed in the far field, one angle at a time. From the perspective of $\mathrm{CS}$, the computations are performed using an excitation that is a random combination of plane waves, and the scattered fields are viewed simultaneously at all angles, with the angle-dependent scattered fields projected onto a random vector. The computational cost of a CS analysis is exactly the same as that of a traditional analysis, for which fixed angles of incidence and scattering are considered. The results presented demonstrated that at minimum the CS computations may be viewed as combining the scattering analysis with a regression model, to yield a smooth and highly accurate representation of the scattered fields as a function of angle, with a resolution far superior than that associated with uniform sampling at the same computational cost. The results also demonstrated that the CS-based results are more accurate than those using uniform sampling and simple linear interpolation.

The work presented here constitutes an introduction of CS to the problem of performing a scattering analysis, and therefore there are more open questions for future research than there are problems solved here. As a sample of open research issues, we discuss the following:

In the CS analysis presented here the basis $\Psi$ was real, and therefore we were required to deal with real signals. Moreover, the CS theory [7] has principally only been developed for real signals $u$. The scattered fields of interest are complex, and therefore to constitute real signals $u$ the real and imaginary parts of the scattered fields were concatenated. It is anticipated that the performance of the CS compression will be enhanced if the basis $\Psi$ is extended to be complex, and if CS theory can be extended to complex signals.

In the context of the MLFMM scattering computations, the CS matrix $\Phi$ was constituted by drawing samples from an underlying random variable. While this framework has been demonstrated to give good results, and is blessed with good theoretical properties [7], it has recently been demonstrated that if the underlying signal $u$ is structured, improved performance may be achieved if the $\Phi$ matrix is constituted adaptively, sequentially [12]. Noting the relatively highly structured form of angle-dependent scattering measurements, it is anticipated that in this context 
the CS-based MLFMM computations may be further accelerated with adaptive formation of $\Phi$ (rather than constituting the $N_{s}$ excitations randomly, they may be constituted adaptively, with a goal of accelerating CS convergence [12].

In the CS analysis one must choose a basis or frame $\Psi$ in which to operate, and it is desirable to pick a $\Psi$ for which the signal of interest is sparsely rendered. Although an exhaustive analysis was not performed, for the problem of interest here it was determined that the discrete cosine transform (DCT) yielded better sparseness than a wavelet basis; this is attributed to the fact that often the angle-dependent scattered fields constitute a very smooth function, and therefore the localizing properties of wavelets are less necessary. An area of interest involves an investigation into a $\Psi$ that is best suited for the scattering analysis of interest. As discussed above, in this context it is expected that $\Psi$ may be complex.

In the analysis presented, we considered a spectral analysis of the fields generated by a source radiating in a complex environment, and demonstrated that a wide range of superposed plane waves may be constituted. This complicated target excitation, and a similar phenomenon for the scattered fields, is responsible for the ability to estimate the angle-dependent scattered fields accurately based on a small set of CS measurements (in situ CS). To simplify the analysis, we only considered real spectral angles, corresponding to a superposition of plane waves. If the surrounding heterogeneity in the CS measurements is sufficiently close to the target, then one may have the opportunity to measure complex (evanescent) spectral components. There are recent time-reversal results that demonstrate the dramatic effects of evanescent fields when the surrounding heterogeneity is close to the target or source [26]; it is therefore of interest to examine whether CS may be applied to measure evanescent fields, with the source and receiver distant from the target (the heterogeneity must be in the near field of the target scattered fields, while the source and receiver antennas need not be in the near field).

Finally, to simplify the analysis presented here, we considered CS measurements at a single frequency, although it was discussed how the basic construct may be extended to estimating the angle and frequency dependent properties of the scattered fields. Moreover, many scattering techniques are implemented in the time domain, such as the FDTD method applied in a subset of the examples considered here. It is of interest to examine how the CS formulation may be 


\section{extended to time-domain scattering measurements ${ }^{2}$.}

\section{REFERENCES}

[1] I. Daubechies, Ten Lectures on Wavelets. SIAM, 1992.

[2] S. Mallat, A Wavelet Tour of Signal Processing, 2nd ed. Academic Press, 1998.

[3] A. Said and W. A. Pearlman, "A new fast and efficient image codec based on set partitioning in hierarchical trees," IEEE Trans. Circuits Systems for Video Technology, vol. 6, pp. 243-250, 1996.

[4] W. A. Pearlman, A. Islam, N. Nagaraj, and A. Said, "Efficient, low-complexity image coding with a set-partitioning embedded block coder," IEEE Trans. Circuits Systems Video Technology, vol. 14, pp. 1219-1235, Nov. 2004.

[5] E. Candès and T. Tao, "The Dantzig selector: statistical estimation when $p$ is much larger than $n$," 2005, Preprint.

[6] E. Candès, J. Romberg, and T. Tao, "Robust uncertainty principles: Exact signal reconstruction from highly incomplete frequency information," IEEE Trans. Information Theory, vol. 52, no. 2, pp. 489-509, Feb. 2006.

[7] D. L. Donoho, “Compressed sensing,” IEEE Trans. Information Theory, vol. 52, no. 4, pp. 1289-1306, Apr. 2006.

[8] J. A. Tropp and A. C. Gilbert, "Signal recovery from partial information via orthogonal matching pursuit," Apr. 2005, Preprint.

[9] D. L. Donoho, Y. Tsaig, I. Drori, and J.-C. Starck, "Sparse solution of underdetermined linear equations by stagewise orthogonal matching pursuit,” Mar. 2006, Preprint.

[10] Y. Tsaig and D. L. Donoho, "Extensions of compressed sensing," Signal Processing, vol. 86, no. 3, pp. 549-571, Mar. 2006.

[11] J. Haupt and R. Nowak, "Signal reconstruction from noisy random projections," IEEE Trans. Information Theory, vol. 52, no. 9, pp. 4036-4048, Sept. 2006.

[12] S. Ji, Y. Xue, and L. Carin, "Bayesian compressive sensing," Jan. 2007, Preprint.

[13] M. Figueiredo, R. D. Nowak, and S. J. Wright, "Gradient projection for sparse reconstruction: Application to compressed sensing and other inverse problems," 2007, Preprint.

[14] M. Fink, “Time reversed acoustics,” Physics Today, vol. 50, pp. 34-40, 1997.

[15] P. Blomberg, G. Papanicolaou, and H. Zhao, "Super-resolution in time-reversal acoustics," J. Acoust. Soc. of Am., vol. 111, pp. 230-248, 2002.

[16] D. Liu, S. Vasudevan, J. Krolik, G. Bal, and L. Carin, "Electromagnetic time-reversal source localization in changing media: Experiment and analysis," IEEE Trans. Antennas Propagation, vol. 55, pp. 344-354, Feb. 2007.

[17] L. Felsen and N. Marcuvitz, Radiation and Scattering of Waves. Piscataway, NJ: IEEE Press, 1994.

[18] A. Devaney, "A filtered backpropagation algorithm for diffraction tomography," Ultrasonic Imaging, vol. 4, pp. 336-360, 1982.

[19] C. Balanis, Advanced Engineering Electromagnetics. New York, NY: Wiley, 1989.

[20] A. Taflove and S. hagness, Computational Electromagnetics: The Finite Difference Time-Domain Method. Artech House, 2005.

[21] J. Berenger, "A perfectly matched layer for the absorption of electromagnetic waves," Journal of Computational Physics, vol. 114, p. 185200, 1994.

[22] R. Harrington, Field Computation by Moment Methods. IEEE Press, 1993.

\footnotetext{
${ }^{2}$ The research reported here was supported by DARPA, under the Mathematical Time Reversal program
} 
[23] R. Coifman, V. Rokhlin, and S. Wandzura, "The fast multipole method for the wave equation: a pedestrian prescription," IEEE Ant. and Prop. Magazine, vol. 35, pp. 7-12, 1993.

[24] J. Song, C. Lu, and W. Chew, "Multilevel fast multipole algorithm for electromagnetic scattering by large complex objects," IEEE Trans. Ant. and Prop., vol. 45, pp. 1488-1493, 1997.

[25] J. He, A. Sullivan, and L. Carin, "Multilevel fast multipole algorithm for three-dimensional dielectric targets in the vicinity of a lossy half space," Microwave and Optical Technology Letters, vol. 29, pp. 100-104, 2001.

[26] G. Lerosey, J. de Rosny, A. Tourin, and M. Fink, "Focusing beyond the diffraction limit with far-field time reversal," Science, vol. 315, p. 1120, 2007. 